\title{
Surgery in the Management of Cancer of the Larynx in Benin
}

\author{
N.E. Okolugbo, M.B.B.S (Senior Resident) and F.O. Ogisi, DLO, FRCS, FMCORL, FWACS
}

\begin{abstract}
This study is a retrospective analysis of the survival results of 33 patients managed surgically for cancer of the larynx in the University of Benin Teaching Hospital, Benin City. Various surgeries carried out include; tracheostomies, direct laryngoscopy and biopsies, and total laryngectomies ( primary / salvage ). It was found that those who primary laryngectomy had less complications after surgery with better chances of survival than those who had salvage laryngectomy.
\end{abstract}

\section{INTRODUCTION}

One of the modalities of managing cancer of the larynx is surgery, the others being radiotherapy and chemotherapy ${ }^{1}$.

The various surgeries that can be done in the management of cancer of the larynx include partial laryngectomy, total laryngectomy and the surgical voice restoration Partial Laryngeal surgery is an option for early stage tumours ${ }^{2}$, while late stage tumors tend to be treated with total laryngectomy usually followed with post operative chemoradiotherapy. Other forms of surgery done include tracheostomy which though not desirable is usually done, a neck dissection depending on the cervical nodal status of the patient ${ }^{3}$, a feeding gastrostomy for palliative treatment of advanced cases, and of course a

KEY WORDS: Laryngeal carcinoma, direct laryngoscopy, Biopsy, Total laryngectamy.

ENT Unit, Department of Surgery, University of Benin Teaching Hospital, Benin City

Correspondence : Dr. N. E. Okolugbo, Dept. of Surgery, U.B.T.H. , Benin City. direct laryngoscopy and biopsy will have to necessarily be done for all patients to obtain a histological diagnosis.

We present in this paper, a five year review of the various surgeries carried out in the University of Benin Teaching. Hospital during the course of managing carcinoma of the larynx. Our aim was to analyse the outcome of those surgeries and highlight factors that could lead to a significant improvement in the prognosis of our patients.

\section{METHODOLOGY}

The case records of all the patient histologically diagnosed to have carcinoma of the larynx between June 1999 and May 2004 in the ORU Clinic of U.B.T.H, Benin City were retrieved and analysed with the aid of frequency distribution table. Data extracted were;

\section{RESULTS}

A total of 33 patients were seen within the period of 5 years (1999 to 2004).

Table 1 showed the age group distribution of the patients with majority (63.7\%) being in the $5^{\text {th }}$ and $6^{\text {th }}$ Decades. 


\section{Table 1: Frequency table showing $\%$ Distribution by Age}

\begin{tabular}{lcc}
\hline Age in Years & Number & Percentage \\
\hline $31-40$ & 3 & $9.1 \%$ \\
$41-50$ & 7 & $21.2 \%$ \\
$51-60$ & 9 & $27.3 \%$ \\
$61-70$ & 12 & $36.4 \%$ \\
$\geq 71$ & 2 & $6.0 \%$ \\
\hline Total & 33 & $100 \%$ \\
\hline
\end{tabular}

These were 29 males and 4 females, giving a male to female ratio of $7.25: 1$

In tumour staging, all patients underwent indirect as well as direct Laryngoscopy. Each patient also had a plain X-ray of the neck as well as a CT scan of the larynx done.

The cervical nodal status was assessed clinically.

About forty-two a half were classified as early stage tumor. While $48.6 \%$ had $\mathrm{T}_{3}$ tumors without palpable nodes and 9\% had $\mathrm{T}_{3}$ tunours with palpable nodes.

\section{Table 3: Stage of Tumours}

\begin{tabular}{|l|l|}
\hline $\mathrm{T}_{1}$ No $\mathrm{Mx}$ & $42.4 \%$ \\
$\mathrm{~T}_{2}$ No Mx. & \\
\hline $\mathrm{T}_{3}$ No Mx & $48.6 \%$ \\
\hline $\mathrm{T}_{3} \mathrm{~N}_{1} \mathrm{Mx}$ & $9 \%$ \\
$\mathrm{~T}_{3} \mathrm{~N}_{2} \mathrm{Mx}$ & \\
\hline
\end{tabular}

The surgeries done in the course of management of the patients were direct laryngoscopy and biopsies, tracheostomies. Total laryngectomies (salvage/primary) and feeding gastrostomies

Direct laryngoscopy and biopsy was done in all cases.

Tracheostomy was done in all but I case. Primary total laryngecotomy was done in 4 cases while 2 patients had salvage total laryngecotomies. 2 of our patients had feeding gastrostomy done.

Post-operative hospitalisation after total laryngectomy ranged from 20 to 43 days with a average of 30.2 days.

\footnotetext{
(. CMS UNIBEN JMBR 2006; 5 (1): 13-15
}

Wound Complications, wound sloughing/ delayed healing was only seen in the 2 patients that had salvage total laryngectomy done. All patients had few swallowing problems following oral intake which was initiated on the $13^{\text {th }}$ day post operatively. The feeding tube was removed between 14 and 21 days post operatively with an average of 16.5 days. Two patients had significant weight loss, while 2 had recurrent chest infections. 2 patients also developed pharyngocutaneous fistula; 1 patient developed hypocalcemia, while post operative hypothyroidism was noted in one patient

One mortality was recorded in the second post operative month of one of the patients that had salvage total laryngectomy.

\section{Table 4: Incidence of complications following total laryngectomy}

\begin{tabular}{|ll|l|}
\hline 1. Delayed wound healing/slough & 2 \\
2. & Swallowing problems & 6 \\
3. & Re-current chest infections & 2 \\
4. & Pharyngocutaneous fistula & 2 \\
5. Hypocalcaemia & 1 \\
6. Hypothyroidism & 1 \\
\hline
\end{tabular}

\section{DISCUSSION}

Treatment of cancer of the larynx involves radiotherapy, surgery or a combination of both surgery and chemo-radiotherapy as adjuvant methods of treatment. 
Generally early stage Tumours $\left(\mathrm{T}_{1} \mathrm{~N}_{\circ}-\right.$ $\mathrm{T}_{2} \mathrm{NO}$ ) are usually treated by radiotherapy and surgery is reserved for recurrence due to failure of radiotherapy or residual disease. ${ }^{1,2}$.

In late stage tumours $\left(\mathrm{T}_{3}-\mathrm{T}_{4}\right)$. Most workers agree that a total laryngectomy in combination with post-operative radiotherapy constitutes optimal treatment. ${ }^{3-5}$

Some other workers have advocated chemotherapy and radiotherapy protocol for stage III and IV carcinoma of the larynx with an attempt at laryngeal preservation and they claim good results ${ }^{6,7,8}$.

However from our experience where most of our patients presented with stage 3 tumours (57.6\%) and some were not favourably disposed to total laryngectomy, results from the radiation therapy, administered to them were not encouraging as though most were lost to follow-up, 5 presented later with varying degrees of tumour recurrence and lymph node metastasis necessitating salvage total laryngectomy.

It was also noted that the more severe complications such as pharyngocutaneous fistula, hypocalcaemia recurrent chest infections were observed in the 2 patients that had salvage laryngectomy.

For patients who had primary laryngectomy, their wounds healed well and there was little or no complications in the immediate postoperative period except for one of them with a $\mathrm{T}_{4}$ Tumour, who developed hypothyroidism. This is in agreement with the findings of Macomb ${ }^{9}$ who reported that the mortality rate of all composite resections about the Head and neck is $4.4 \%$ without pre-operative irradiation and $8.5 \%$ after pre operative irradiation.

\section{CONCLUSION}

It was found that those who had primary laryngectomy had less complications after surgery with better chances at survival.

\section{References}

1. Staphshay, S M. Hybets R U Treatment of Cancer of the Larynx. . Otolaryngologic Clinics of North Africa 1995; 18: 461-468.

2. Hibbs, G G. Ying, D and Hendrickson F R. Radiotherapy for early stages of vocal cord cancer. Ann. Otol Rhinol Laryngol 1969;78: 319.

3. Bryce, D P. Ireland P E. and Rider, W D. Experience in the Surgical and radiological treatment in 500 cases of carcinoma of the larynx. Ann. Otol. Rhinol. Laryngol, 1963; 72: 416 .

4. Putney F J. and chapman C E. Carcinoma of the larynx, analysis of 311 cases treated surgically. Ann Otol Rhinol Laryngol. 1972; 81:445,

5. Wang. C C., Schulz, M D. Miler D. Combined Radiation Therapy and surgery for carcinoma of the supraglottis and pyriform sinus. Laryngoscope 1972; 82: 1883.

6. Bahadur et al. Laryngeal preservation with induction chemotherapy and Radiotherapy protocol for stage III and IV carcinoma of the larynx - results of a pilot study. The journal of laryngology \& otology 1999; 113 : 233-437.

7. Kazem I, Van de brook P. Planned preoperative radiation therapy Vs definitive radiotherapy for advanced laryngeal carcinoma. Laryngoscope 1984; 94 : 1355 1358.

8. Vikram B. Farr H W. Adjuvant irradiation therapy for locally advanced head and neck cancer. Cancer 1983; 33: 134-138.

9. Maccomb W S. : Mortality from radical neck dissection. Amer. J. Surgery 1968;115:342. 